\title{
Pituitary metastases: current practice in Japan
}

\author{
Mika Habu, MD, ${ }^{1}$ Hiroshi Tokimura, MD, PhD, ${ }^{1}$ Hirofumi Hirano, MD, PhD, ${ }^{1}$ \\ Soichiro Yasuda, MD, ${ }^{2}$ Yasushi Nagatomo, MD, PhD, ${ }^{3}$ Yoshiyasu Iwai, MD, PhD, ${ }^{4}$ \\ Jun Kawagishi, MD, PhD, ${ }^{5}$ Koshi Tatewaki, MD, PhD, ${ }^{6}$ Shunji Yunoue, MD, PhD, ${ }^{1}$ \\ Francia Campos, MD, PhD, ${ }^{1}$ Yasuyuki Kinoshita, MD, PhD, ${ }^{7}$ Akira Shimatsu, MD, DMS, ${ }^{8}$ \\ Akira Teramoto, MD, PhD, ${ }^{9}$ and Kazunori Arita, MD, PhD ${ }^{1}$
}

\begin{abstract}
${ }^{1}$ Department of Neurosurgery, Graduate School of Medical and Dental Sciences, Kagoshima University, Sakuragaoka, Kagoshima; ${ }^{2}$ Department of Neurosurgery, Shiroyama Hospital, Habikino; ${ }^{3}$ Department of Neurosurgery, Kouseikai Takai Hospital, Tenri; ${ }^{2}$ Department of Neurosurgery, Osaka City General Hospital, Osaka; ${ }^{5}$ Jiro Suzuki Memorial Gamma House, Furukawa Seiryo Hospital, Osaki; ${ }^{6}$ Yokohama CyberKnife Center, Shinryoku Neourosurgical Clinic, Yokohama; ${ }^{7}$ Department of Neurosurgery, Graduate School of Biomedical and Health Sciences, Hiroshima University, Hiroshima; ${ }^{8}$ Clinical Research Institute, National Hospital Organization Kyoto Medical Center, Kyoto; and ${ }^{9}$ Tokyo Rosai Hospital, Tokyo, Japan
\end{abstract}

\begin{abstract}
OBJECT With advancement of cancer treatment and development of neuroimaging techniques, contemporary clinical pictures of pituitary metastases (PMs) must have changed from past reports. The goal of this paper was to elucidate the clinical features of PMs and current clinical practice related to those lesions. In this retrospective study, questionnaires were sent to 87 physicians who had treated PMs in Japan.

RESULTS Between 1995 and 2010, 201 patients with PMs were treated by the participating physicians. The diagnosis of PM was histologically verified in 69 patients (34.3\%). In the other 132 patients $(65.7 \%)$, the PM was diagnosed by their physicians based on neuroimaging findings and clinical courses. The most frequent primary tumor was lung (36.8\%), followed by breast $(22.9 \%)$ and kidney $(7.0 \%)$ cancer. The average interval between diagnosis of primary cancer and detection of PM was $2.8 \pm 3.9$ (SD) years. Major symptoms at diagnosis were visual disturbance in $30.3 \%$, diabetes insipidus in $27.4 \%$, fatigue in $25.4 \%$, headache in $20.4 \%$, and double vision in $17.4 \%$. Major neuroimaging features were mass lesion in the pituitary stalk (63.3\%), constriction of tumor at the diaphragmatic hiatus (44.7\%), hypothalamic mass lesion $(17.4 \%)$, and hyperintensity in the optic tract (11.4\%). Surgical treatment was performed in $26.9 \%$ of patients, and $74.6 \%$ had radiation therapy; $80.0 \%$ of patients who underwent radiotherapy had stereotactic radiotherapy. The median survival time was 12.9 months in total. Contributing factors for good prognosis calculated by Cox proportional hazard analysis were younger age, late metastasis to the pituitary gland, smaller PM size, and radiation therapy. The Kaplan-Meier survival was significantly better in patients with breast cancer and renal cell cancer than in those with lung cancer.
\end{abstract}

CONCLUSIONS At the time of this writing, approximately $60 \%$ (120/201) of PMs had been treated by stereotactic radiation therapy in Japan. The median survival time was much longer than that reported in past series. To confirm the changes of clinical features and medical practice, a prospective and population-based survey is mandatory.

http://thejns.org/doi/abs/10.3171/2014.12.JNS14870

KEY WORDS pituitary metastasis; MRI; symptom; survival time; prognostic factor; oncology

A LTHOUGH malignant tumors metastasizing into the pituitary fossa are not infrequent events in autopsy series, ${ }^{6,10,17,18}$ pituitary metastasis (PM) is rarely diagnosed ante mortem. ${ }^{3,14,18}$ According to the Brain Tumor Registry of Japan, only $0.4 \%$ of intracranial metastatic tumors are located in the pituitary gland..$^{5}$ These tumors account for only $1 \%$ of surgically treated pituitary tu- mors. ${ }^{8,16,31}$ Recently, as the number of patients diagnosed with cancer has increased and their survival time has been extended, PMs have been diagnosed more frequently than before. ${ }^{16,20,24}$

Many authors have reported their own experiences or their institutional series on PMs., 3,11,13,14,20,26,31 There are also reviews involving large numbers of reported cas-

\section{ABBREVIATION PM = pituitary metastasis.}

SUBMITTED April 18, 2014. ACCEPTED December 30, 2014.

INCLUDE WHEN CITING Published online July 17, 2015; DOI: 10.3171/2014.12.JNS14870.

DISCLOSURE This study was financed by the National Cancer Center Research and Development Fund (grant no. 21-8-6: "Diagnosis and Treatment of Intractable Endocrine Tumors" by principal investigator, Dr. Akira Shimatsu) and supported by a Health and Labor Sciences Research grant on "Research on Intractable Diseases in Japan: Hypothalamo-Pituitary Dysfunction," and by a grant-in-aid (no. 20591716) awarded to Dr. Kazunori Arita from the Ministry of Education, Culture, Sports, Science, and Technology of Japan. The authors declare that there is no conflict of interest that could be perceived as prejudicing the impartiality of the paper reported. 
es. 4,11,18,20,22,28 Accordingly, prognosis is generally poor, with a median survival time of around 6 months. ${ }^{4,11,18,20,22,27}$ However, these reports and reviews may have been biased from reports of infrequent clinical presentations of patients with this rare disorder ${ }^{16}$ and would be obsolete because some of the cases were treated 3-4 decades ago. Cancer treatments have recently been developing rapidly, and the prognosis for cancer patients has been improving (http:// ganjoho.jp/professional/statistics/). Magnetic resonance imaging has facilitated the early detection of sellar lesions, and the diagnosis of PMs has been reported to largely depend on these imaging studies..$^{13,14}$ Furthermore, recent reports have depicted cases of patients who survived long after the diagnosis..$^{13,31}$ We should, therefore, consider the lack of a clear clinical picture of this disease in the era of MRI and advanced cancer treatment, which are essential for proper management of this disease. Therefore, we conducted a questionnaire study to elicit the diagnostic practice, clinical and radiological features, treatment modalities, and prognosis of PMs treated during a recent 15-year period in Japan.

\section{Methods}

\section{Survey by Questionnaire}

The first questionnaire simply asked about physicians' experiences with treating patients with PMs during a 15year time period (1995-2010). The first questionnaire was sent by mail to all 365 clinics that have been designated as training centers for clinical endocrinology by the Japan Endocrine Society, which covers most endocrinology clinics in Japan, and to 860 members of The Japanese Society for Hypothalamic and Pituitary Tumors, representing a majority of centers treating hypothalamopituitary diseases in Japan. Considering the recent trend in Japan that general internists and surgeons treating cancers frequently send patients to stereotactic radiation centers without consulting neurosurgeons or endocrinologists when they find intracranial metastasis, the questionnaire was also sent to 56 stereotactic radiotherapy centers. The first questionnaire was distributed to 1069 physicians in 626 hospitals. Four hundred twenty-three $(39.6 \%)$ physicians from 314 $(50.2 \%)$ hospitals replied to the questionnaire; among them, 20.6\% (87/423) from 84 hospitals reported that they had treated patients with PMs within the 15-year time span. Reports of 209 PMs were collected through the first questionnaire.

Subsequent questionnaires were sent to these 87 physicians to obtain detailed information, such as age, sex, primary tumors, symptoms, endocrinological status, MRI findings, treatment modalities such as surgery and radiotherapy, complications, and prognosis.

To determine the characteristics of extension of PMs, we compared geometric indicators on MRI of PMs with those of previously reported 50 cases of clinically nonfunctioning pituitary adenoma treated in Kagoshima University Hospital during 2007-2011. ${ }^{1}$

\section{Statistical Analyses}

The Statflex version 6.0 software program (Artech Co.) was used for statistical analysis of the results. Depending on the characteristics of the data sets, data were analyzed using Fisher's exact test, the Mann-Whitney U-test, Student's test, or a simple correlation test. Mean values are reported as the mean \pm SD. Differences of $p<0.05$ were considered statistically significant. The Kaplan-Meier method and Cox proportional hazard model were used for survival analyses.

\section{Ethical Considerations}

This retrospective study was approved by the Ethics Committee of Kagoshima University Hospital. We certify that this study involving human subjects is in accordance with the Helsinki Declaration of 1975 as revised in 2000 and with the Ethical Guidelines for Epidemiological Research (effective on July 1, 2002) by the Ministry of Education, Culture, Sports, Science and Technology, and Ministry of Health, Labor and Welfare, Japan. To protect patient privacy, all data were collected and analyzed after anonymization in an unlinkable fashion.

\section{Results \\ Demography}

Scrupulous collation found that 8 cases were reported twice from different institutions. Therefore, a total of 201 patients with PMs were included in this study (male/female 109:92). The patients' ages ranged from 26 to 85 years with a mean of $59.0 \pm 11.4$ years (error rate $1.4 \%$ ). The age of 1 patient was not reported. The most common age group was the 60- to 64-year group, followed by the 55- to 59-year group (Fig. 1). The average age of the 108 male patients was $61.1 \pm 10.7$ years (error rate $1.7 \%$ ), and that of the 92 female patients was $56.5 \pm 11.7$ years (error rate $2.7 \%$ ).

\section{Diagnosis of PM}

It was noteworthy that the diagnosis of PM was histologically verified in only 69 patients $(34.3 \%)$. These patients were classified as the "definite group." In the other 132 patients $(65.7 \%)$, the PM was diagnosed by their physicians-in-charge based on neuroimaging findings, clinical presentation, clinical course, and an increase in blood tumor markers, and combinations of these factors. These

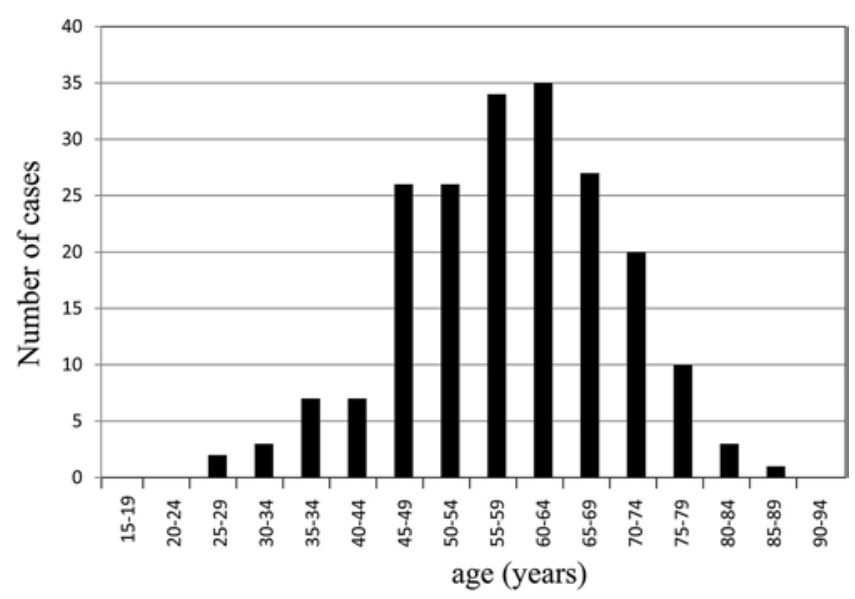

FIG. 1. Age distribution of patients with pituitary metastases $(n=200)$. Age was not listed for 1 patient. The majority (83.6\%) of patients were between 45 and 74 years of age. 
nonhistologically verified tumors were classified as the "nondefinite group."

\section{Primary Tumor Sites}

In the 201 patients, the most frequent primary tumor was lung cancer (36.8\%), followed by breast (22.9\%), kidney (7.0\%), and colorectal (6.5\%) cancer (Fig. 2). Among 109 men, lung cancer was the most frequent primary tumor $(45.9 \%)$, followed by kidney (11.0\%), lymphatic (8.3\%), liver (7.3\%), and colorectal (4.6\%) cancer (Fig. 3 left). Among 92 women, breast cancer was the most frequent $(50.0 \%)$, followed by lung (26.1\%) and colorectal (8.7\%) cancer (Fig. 3 right).

\section{Interval Between the Diagnosis of Primary Tumor and PM}

The interval between detection of the PM and primary tumor was reported in 157 cases. Detection of the pituitary lesion preceded the diagnosis of primary tumor in 17 of 157 cases $(10.8 \%)$. In the remaining 140 patients, the average interval between diagnosis of the initial cancer and detection of the PM was $2.8 \pm 3.9$ years (error rate $11.8 \%$ ). The interval was within 1 year in $45.0 \%(n=63), 1-3$ years in $22.9 \%(\mathrm{n}=32), 3-5$ years in $12.1 \%(\mathrm{n}=17), 5-10$ years in $15.0 \%(\mathrm{n}=21)$, and longer than 10 years in $5.0 \%(\mathrm{n}=7)$ of the 140 patients. In 63 patients with a PM detected within 1 year after the diagnosis of primary tumor, the most frequent primary tumor was lung cancer $(61.9 \%)$ followed by lymphoma (7.9\%). On the contrary, among a total of 28 patients whose pituitary lesion was detected more than 5 years after the diagnosis of primary tumor, the most frequent primary tumor was breast cancer $(53.6 \%)$ followed by colorectal (10.7\%) and kidney (10.7\%) cancer. Only $7.1 \%$ of this group of patients had lung cancer.

\section{Symptoms}

Symptoms at the time of diagnosis of PMs greatly varied, with visual disturbance in $30.3 \%$, diabetes insipidus in $27.4 \%$, fatigue in $25.4 \%$, headache in $20.4 \%$, double vision in $17.4 \%$, nausea/vomiting in $11.4 \%$, consciousness disturbance in $8.0 \%$, appetite loss in $6.5 \%$, seizure in $1.5 \%$, and cold intolerance in $1.0 \%$ of total cases (multiple answers were allowed for this question) (Fig. 4). It was noticeable that symptoms that are rarely seen in pituitary adenoma such as diabetes insipidus and double vision were not uncommon in this PM series.

\section{Anterior Pituitary Functions}

Hormone assays varied among institutions, and the assessment of pituitary function was left to the discretion of the participating physicians. Patients with hypoadrenocorticism, as judged by low serum cortisol levels or newly initiated hydrocortisone replacement, accounted for $42.3 \%$ $(85 / 201)$ of all and those with hypothyroidism, as judged by low serum thyroxine levels or newly initiated levothyroxine replacement, accounted for $43.3 \%$ (87/201) of all. Among 54 patients whose insulin-like growth factor level was measured, 26 (48.1\%) showed low levels, less than -2 standard deviations of the age- and sex-matched normal population. The gonadal function was not included in the questionnaire due to the difficulty of judging its function solely by the levels of sex steroids.

\section{Neuroimaging Findings}

All patients underwent MRI. Mass lesions in other intracranial sites were seen in $45.0 \%$ (81/180; denominator in this paragraph is the total number of responses to each particular question [Table 1]). Among 180 patients who

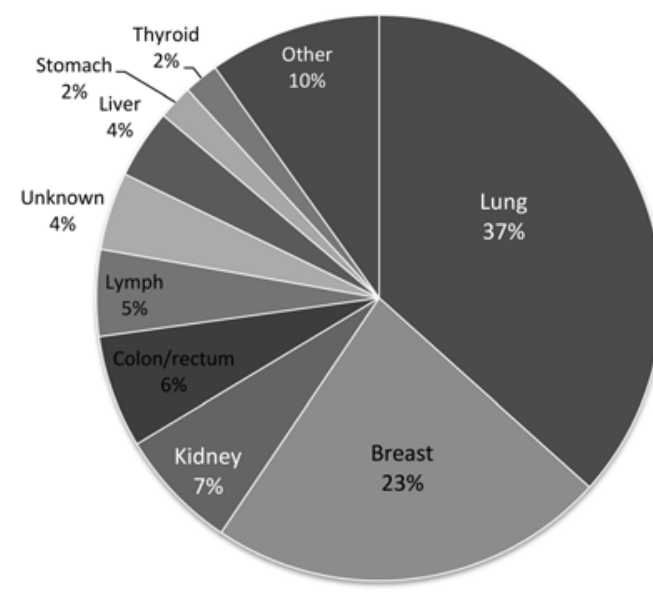

\begin{tabular}{|c|c|c|}
\hline Origin & Numbers & $\%$ \\
\hline Lung & 74 & 36.8 \\
\hline Breast & 46 & 22.9 \\
\hline Kidney & 14 & 7.0 \\
\hline Colon/rectum & 13 & 6.5 \\
\hline Lymph & 10 & 5.0 \\
\hline Unknown & 9 & 4.5 \\
\hline Liver & 8 & 4.0 \\
\hline Stomach & 4 & 2.0 \\
\hline Thyroid & 4 & 2.0 \\
\hline Larynx/pharynx & 3 & 1.5 \\
\hline Parotid gland & 3 & 1.5 \\
\hline Thymus & 2 & 1.0 \\
\hline Blood & 2 & 1.0 \\
\hline Esophagus & 2 & 1.0 \\
\hline Gallbladder/bile duct & 1 & 0.5 \\
\hline Adrenal gland & 1 & 0.5 \\
\hline Uterine tube & 1 & 0.5 \\
\hline Bladder & 1 & 0.5 \\
\hline Pancreas & 1 & 0.5 \\
\hline Prostate & 1 & 0.5 \\
\hline Retroperitoneum & 1 & 0.5 \\
\hline
\end{tabular}

FIG. 2. Distribution of primary tumor site in total $(n=201)$. 


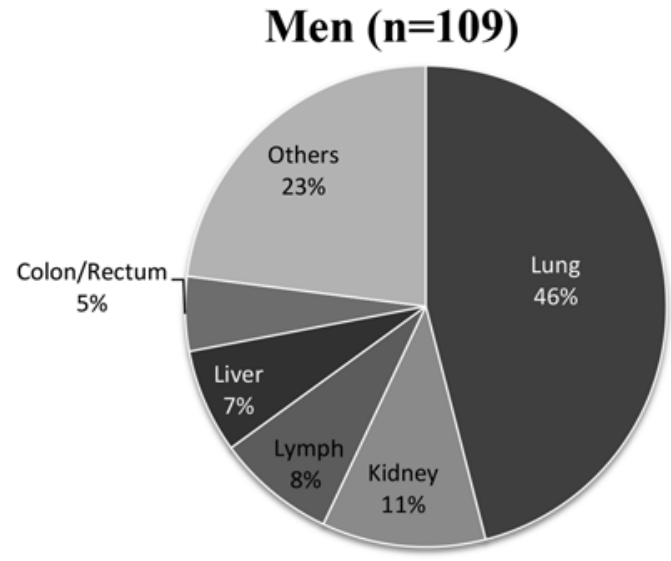

FIG. 3. Distribution of primary tumor by sex.

underwent MRI with gadolinium contrast, 176 (97.8\%) tumors were well enhanced, while $4(2.2 \%)$ tumors were poorly enhanced. The incidence of MRI findings, according to the physicians or radiologists who evaluated the images, were as follows: mass lesion in the pituitary stalk, $63.7 \%$ (107/168); dumbbell shape or constriction of tumor at diaphragmatic hiatus (Fig. 5), 44.7\% (80/179); loss of hyperintensity in neurohypophysis, $82.3 \%$ (107/130); contrast enhancement of dura mater around the pituitary fossa, $17.1 \%$ (29/170); hypothalamic mass lesion, 17.4\% (30/172); hyperintensity in the optic tract with or without the internal capsule (Fig. 6), 11.5\% (17/148); and intratumoral bleeding, $8.5 \%(15 / 176)$.

\section{Size and Direction of Extension in PM}

The size of the PMs, reported in 185 cases, ranged from 1 to $60 \mathrm{~mm}$ with mean of $20.9 \pm 10.6 \mathrm{~mm}$ (error rate $3.7 \%$ ). The mean length of upward extension of the tumor (Fig. 7), that is, the distance of uppermost part of tumor from the line connecting tuberculum sellae and tip of dorsum sellae (TD line), was $12.8 \pm 8.6 \mathrm{~mm}$ (error rate $4.9 \%$ ). The mean length of upward extension of PMs was longer than that of 50 nonfunctioning pituitary adenomas $(10.3 \pm 6.5 \mathrm{~mm}$, error rate $8.9 \%$ ), which we previously reported, ${ }^{1}$ but statistically insignificant ( $p=0.12$, Mann-Whitney U-test). The length of downward extension (Fig. 8), the distance of the lowermost part of the tumor from the standardized sellar floor, which is $7.2 \mathrm{~mm}$ deeper from the TD line, ${ }^{1,30}$ was 3.0

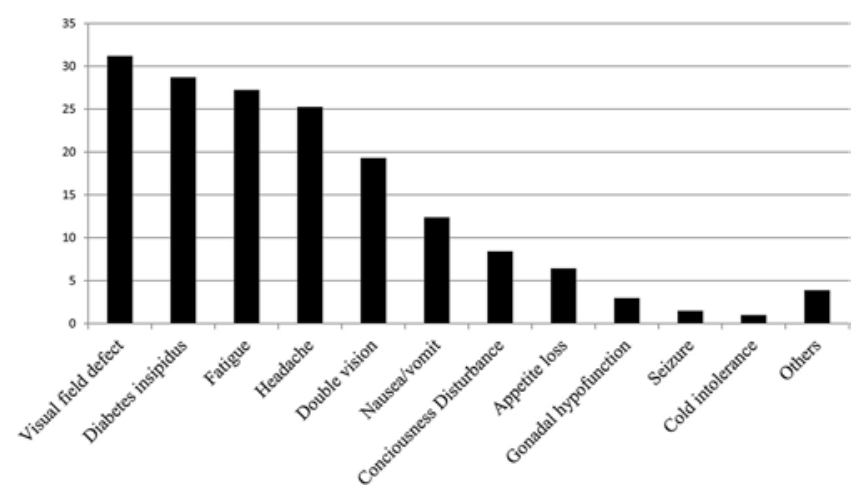

FIG. 4. Frequency of clinical symptoms. Values on the y axis represent the percentage of patients.

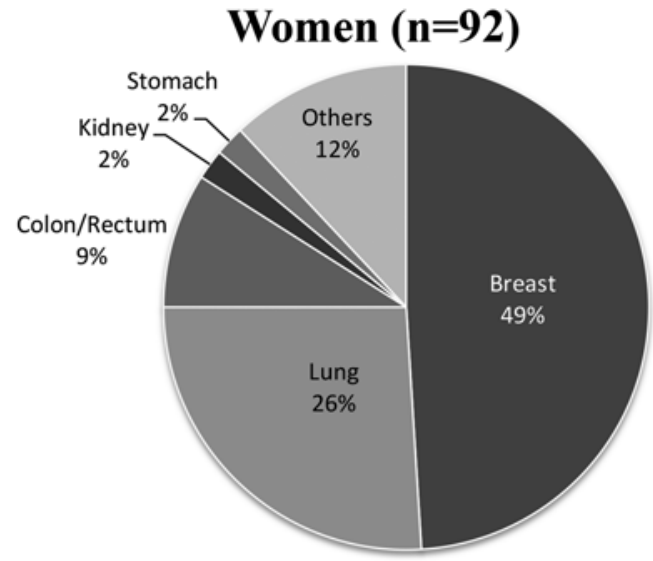

$\pm 3.9 \mathrm{~mm}$ (error rate $9.6 \%$ ), which was significantly shorter than that of 50 nonfunctioning adenomas $(8.9 \pm 4.5 \mathrm{~mm}$, error rate $7.2 \%)^{1}(\mathrm{p}<0.01$, Mann-Whitney U-test) (Fig. 8).

\section{Control of Primary Tumor}

The status of primary tumor control was available in 162 patients. At the time of diagnosis of PM, the primary lesion was thought to be under control in $37.0 \%(60 / 162)$ of the reported patients.

\section{Treatment of PM}

As for treatment, surgery was performed in $26.9 \%$ (54/201) of the patients and radiotherapy targeting the PM was performed in $74.6 \%(150 / 201)$ of total patients. Of these 150 patients, $80.0 \%$ (120/150) underwent stereotactic radiotherapy including Gamma Knife in 108 patients, CyberKnife in 11 patients, and stereotactic linear accelerator in 1 patient. For Gamma Knife surgery, a median peripheral dose of 18.0 Gy was given in most cases in a single session, with an average dose of $16.7 \pm 4.8 \mathrm{~Gy}$. Two or three sessions of Gamma Knife surgery were performed in 3 patients. For CyberKnife treatment, a median peripheral x-ray dose of 30 Gy was given in 3-8 fractions, with an average dose of $28.0 \pm 5.9 \mathrm{~Gy}$. External beam xray radiation was given in 30 patients with a median dose of $40 \mathrm{~Gy}$ in 18 fractions. In 6 patients, the second stereo-

TABLE 1. Findings on MRI

\begin{tabular}{lcc}
\hline \multicolumn{1}{c}{ Finding } & $\begin{array}{c}\text { No. of Answers } \\
\text { to the Question }\end{array}$ & $\begin{array}{c}\text { No. of Patients } \\
\text { w/ Finding (\%) }\end{array}$ \\
\hline Other intracranial lesions & 180 & $81(45.0)$ \\
\hline Hypothalamic mass & 172 & $30(17.4)$ \\
\hline Mass in pituitary stalk & 168 & $107(63.7)$ \\
\hline $\begin{array}{l}\text { Loss of hyperintensity of neurohy- } \\
\text { pophysis }\end{array}$ & 130 & $107(82.3)$ \\
$\begin{array}{l}\text { Hemorrhage in the tumor } \\
\begin{array}{l}\text { Constriction of tumor at diaphrag- } \\
\text { matic hiatus }\end{array}\end{array}$ & 176 & $15(8.5)$ \\
\hline $\begin{array}{l}\text { Hyperintensity around optic tract } \\
\text { Contrast enhancement of surround- } \\
\text { ing dura mater }\end{array}$ & 148 & $80(44.7)$ \\
\hline
\end{tabular}




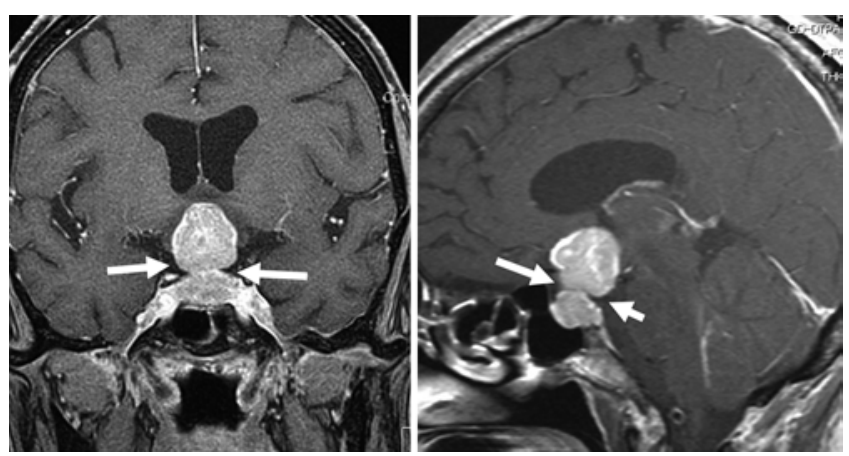

FIG. 5. Coronal (left) and sagittal (right) MR images of a histologically verified pituitary metastasis from lung cancer showing a constriction at the diaphragmatic hiatus (arrows) of tumor leading to the dumbbell shape.

tactic radiation session was performed using the Gamma Knife or CyberKnife for recurrence of the lesion.

Chemotherapy was instituted in $9.0 \%(18 / 201)$ of the patients after the diagnosis of PM. Treatment targeted to the PM was not given in $15.4 \%$ (31/201) of patients.

As for treatment complications, surgical complications were documented in 5 patients $(9.3 \%)$ among 54 surgeries: 3 in transsphenoidal surgery and 2 in transcranial surgery. Surgical morbidity included hypopituitarism, diabetes insipidus, visual disturbance, and consciousness disturbance. Radiation-induced morbidity was reported in 10 patients $(6.7 \%)$ among 150 patients who underwent initial radiotherapy. These included emesis, hypopituitarism, diabetes insipidus, and visual disturbance.

Consecutive changes in tumor size of PMs for 1 year after radiation therapy were reported in 73 patients. Tumor size decreased in $78.1 \%$ (57/73), increased in $9.6 \%$ (7/73), and was unchanged in $12.3 \%$ (9/73) during the first 6 months. In 7 patients, the tumor size increased after the initial decrease during 1 year after the radiation therapy.

\section{Mortality and Cause of Death}

During the follow-up time ranging from 0.3 to 125.3 months with a mean of $14.0 \pm 19.6$ months (error rate 10.9\%), among 165 patients whose prognosis was reported $91(55.2 \%)$ died. The cause of death was progression of the primary tumor in $51.6 \%$ (47/91), extracranial metastatic lesion in $17.6 \%$ (16/91), intracranial extrapituitary metastatic lesions in 5.5\% (5/91), aggravation of the pituitary lesion in $13.2 \%(12 / 91)$, and not specifically reported in $1.1 \%(1 / 91)$.

\section{Survival Analysis}

For the survival analysis, detailed information of 165 patients was collected. The overall median survival time was 12.9 months. Survival rates at 1,2, and 3 years were $50.9 \%, 32.4 \%$, and $28.2 \%$, respectively (Fig. 9 ).

The median survival time largely varied depending on primary tumor: 8.9 months in 58 patients with lung cancer, 25.6 months in 41 patients with breast cancer, and 33.4 months in 13 patients with renal cancer (Fig. 10A). The Kaplan-Meier survival curve showed significantly better survival in patients with breast cancer and renal cell cancer than in those with lung cancer $(\mathrm{p}=0.0012$, log-rank test).

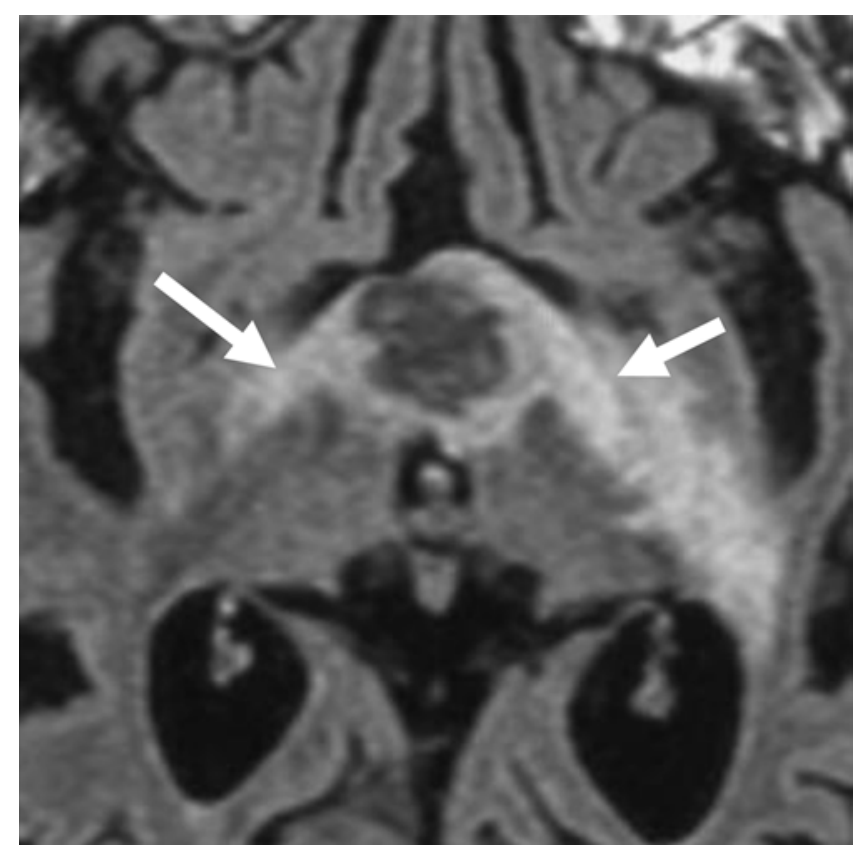

FIG. 6. Fluid attenuated inversion recovery MR image of a histologically verified pituitary metastasis from lung cancer showing hyperintensity alongside the optic tract (arrows).

The status of control of primary tumor affected the survival. The median survival time of patients with good control of primary tumor $(n=52)$ was 46.2 months and that of patients with poor control $(n=86)$ was only 10.2 months; the difference is statistically significant $(\mathrm{p}=0.0014, \log$ rank test) (Fig. 10B).

The survival rate of patients older than 70 years was significantly poorer than that of younger population, with median survival times of 16.8 months in 40 patients 25 49 years old, 18.3 months in 45 patients $50-59$ years old, 9.1 months in 55 patients $60-69$ years old, and 6.9 months in 25 patients $70-85$ years old ( $\mathrm{p}=0.0059, \log$-rank test) (Fig. 10C). The survival rate for women tended to be better than that for men $(p=0.0809)$, with median survival times of 16.8 and 9.1 months in 76 women and 89 men, respectively.

In 123 patients treated with radiation therapy, the median survival time was significantly better (16.4 months) than in 42 patients not treated with radiation $(6.4$ months; $\mathrm{p}=0.0001$ ) (Fig. 10D). The median survival time in 24 patients who underwent conventional multifractionated external beam radiotherapy was 16.1 months and that in 97 patients who underwent stereotactic radiation was 18.7 months; the survival curves were almost overlapped ( $\mathrm{p}=$ 0.743, log-rank test).

\section{Clinical Features of Definite Group and Nondefinite Group}

To elucidate the clinical features of 132 patients whose tumors were not histopathologically diagnosed (the nondefinite group), the following comparisons were conducted (Table 2). In the nondefinite group, the lung was the site of the primary tumor in $43.9 \%$ (58/132) of nondefinite cases and in 23.2\% (16/69) of definite cases. Diagnosis of the PM preceded that of the primary lesions in $6.1 \%$ 

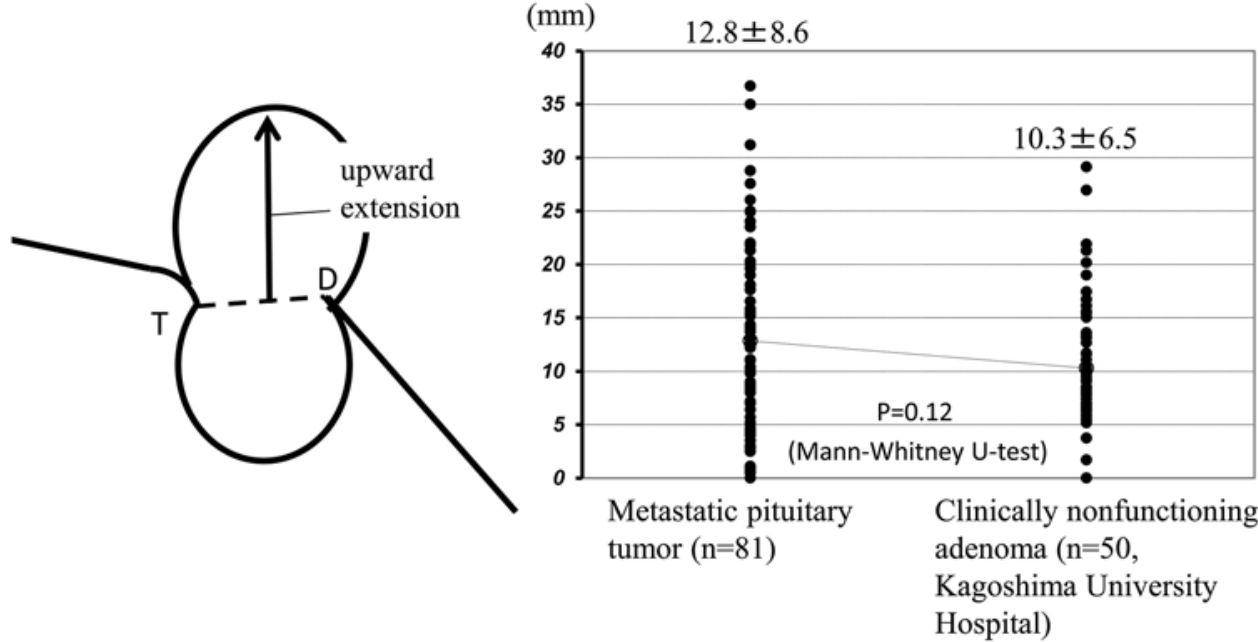

FIG. 7. Upward extension of pituitary metastasis in comparison with clinically nonfunctioning adenoma. $D=$ dorsum sellae; $T=$ tuberculum sellae.

$(8 / 132)$ of cases in the nondefinite group and $13.0 \%(9 / 69)$ of cases in the definite group. The interval until the detection of the PM was less than 1 year in $37.4 \%(49 / 132)$ of patients in the nondefinite group and in $20.3 \%(14 / 69)$ of patients in the definite group. The mean interval was significantly shorter in the nondefinite group than in the definite group $(2.0 \pm 3.2$ years [error rate $17.7 \%$ ] vs $3.5 \pm$ 4.6 years [error rate $16.0 \%$ ]; $p=0.0183$, Student t-test). The presence of other intracranial lesions was more frequently observed in the nondefinite group (47.0\% [62/132]) than in the definite group (27.5\% [19/69]) at the time of diagnosis of PM. The survival time was marginally shorter in the nondefinite group than in the definite group $(\mathrm{p}=0.895$, log-rank test), with a median survival time of 11.4 months versus 13.8 months.

\section{Contributing Factors on Survival Rate}

For Cox proportional hazard analysis, 133 sets of data were available with information regarding age, sex, interval between the diagnosis of primary tumor and PM, control status of the primary lesion, maximum diameter of the
PM, presence of other intracranial lesions, chemotherapy, and radiotherapy (Table 3). In summary, the following were independent factors for better prognosis (hazard ratio $<1, \mathrm{p}<0.05)$ : young age, late metastasis to the pituitary gland, smaller size of the PM, and radiation therapy. Control state of the primary lesion tended to affect the survival $(\mathrm{p}=0.0507)$.

\section{Discussion}

We conducted a nationwide questionnaire survey covering almost all endocrinology and neurosurgery institutions as well as stereotactic radiation centers in Japan to characterize the current clinical features of PMs. Through this study, some contemporary aspects of this disease were elicited.

\section{Clinical Features of PMs}

The most frequent primary tumor of symptomatic PM has been reported to be breast cancer. ${ }^{16,19,20,28}$ However, in this survey, overall the most frequent primary tumor was

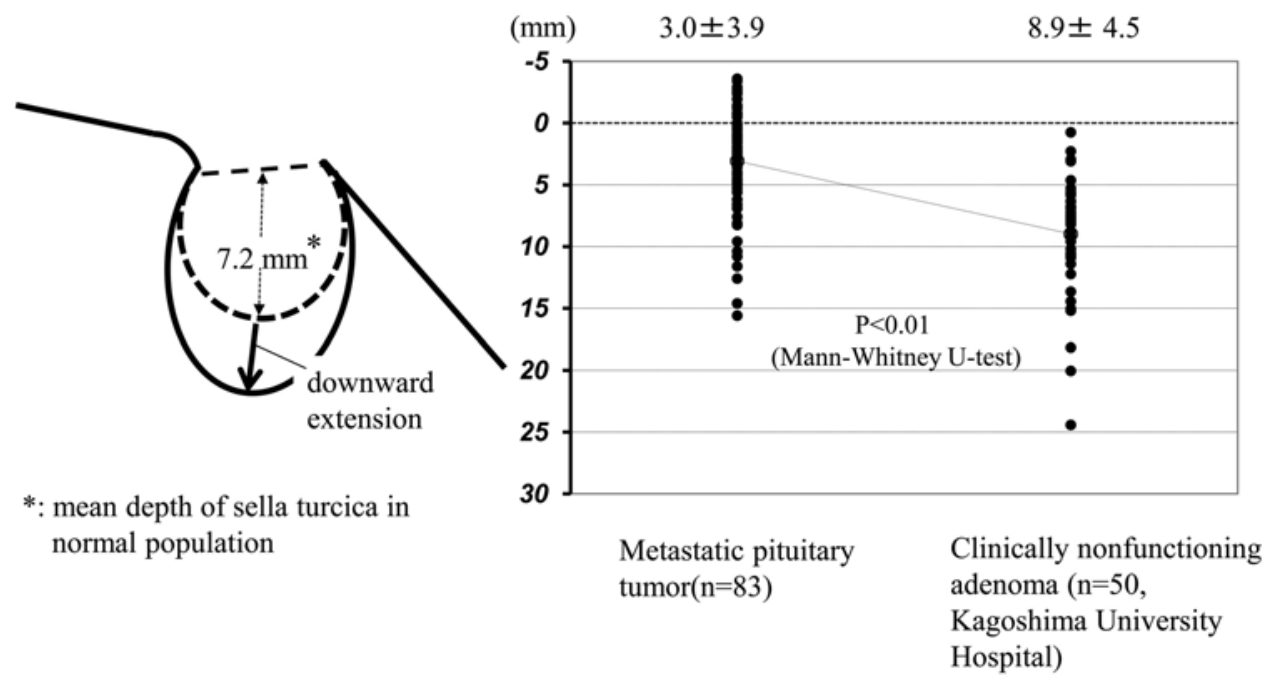

FIG. 8. Downward extension of pituitary metastasis in comparison with clinically nonfunctioning adenoma. 


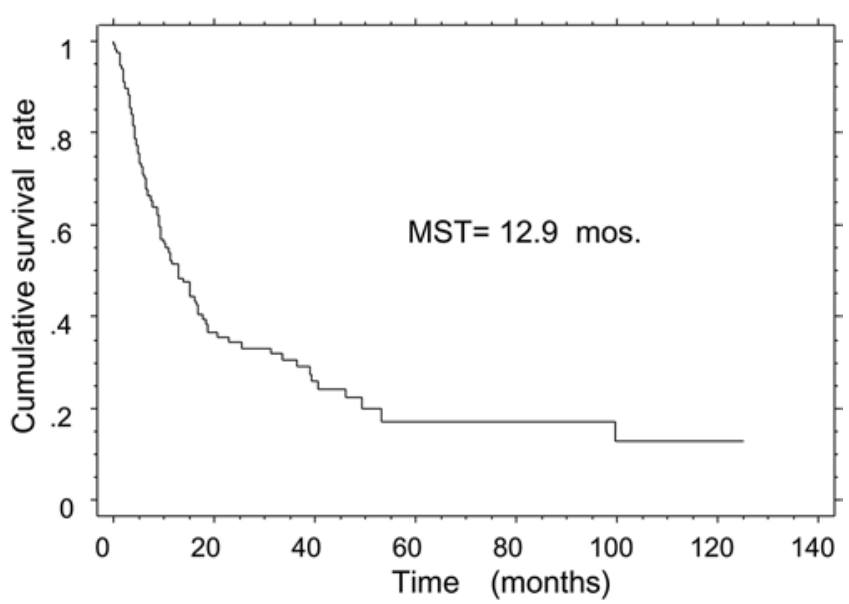

FIG. 9. Survival curve (Kaplan-Meier) of total reported patients $(n=$ 165). MST = median survival time.

lung cancer $(36.8 \%)$, followed by breast cancer $(22.9 \%)$, although breast cancer was still the most frequent $(50.0 \%)$ in women. It is also noteworthy that the incidence of prostate cancer, which was generally reported as the third primary tumor in previous series, ${ }^{7,16}$ was as low as $0.5 \%$ in our series. There were no cases of melanoma in this series.

\section{A. Primary tumors $(\mathrm{N}=112)$}

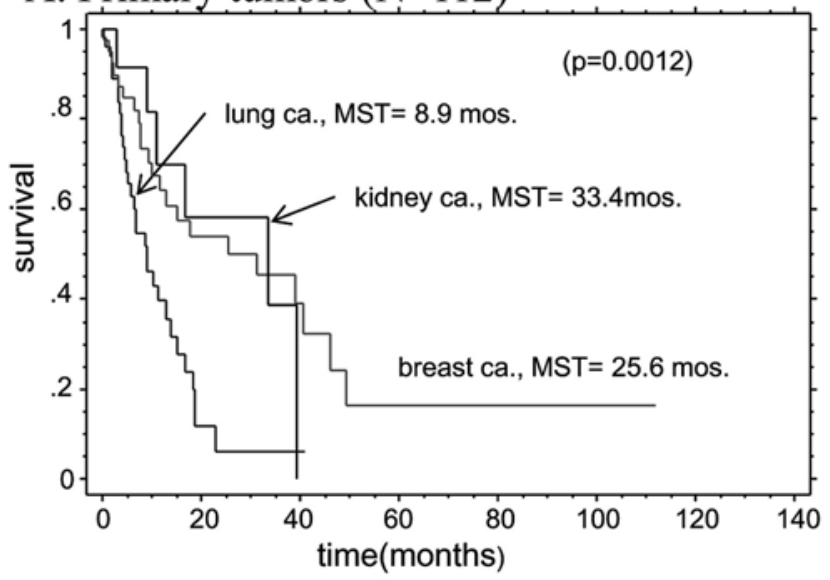

C. Patients' age $(\mathrm{N}=165)$

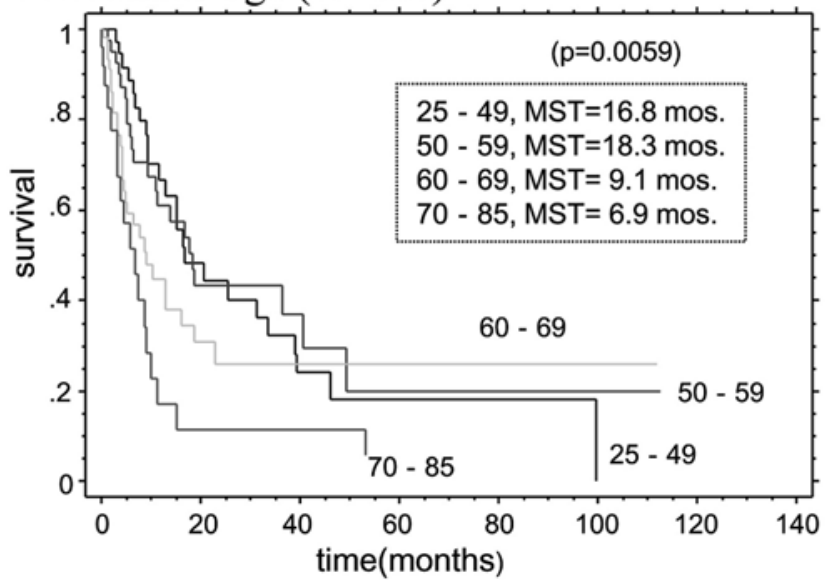

These findings may be a reflection of the relatively low prevalence of these cancers in the Japanese population or in Mongolia. ${ }^{2,12,29}$

A review of 190 symptomatic PMs by Komninos et al. showed that in $83(43.7 \%)$ cases the primary tumor was latent when the PM was discovered. ${ }^{16}$ This figure might be influenced by the multiple reports describing rare presentations. ${ }^{16}$ In our series, primary tumors were generally diagnosed before the detection of PMs; the pituitary lesions were detected before the original tumor in only $10.8 \%$ of cases. The mean intervals for detection of PM widely varied depending on the primary tumor: 0.68 years for lung cancer, 2.84 years for kidney cancer, and 4.76 years for breast cancer. Therefore, the usual 5-year limit could not be set to preclude the possibility for less aggressive tumor such as breast or colorectal cancer to metastasize into the pituitary gland.

\section{Imaging Diagnosis}

Pituitary adenoma is benign and the most common sellar tumor, which accounts for $76.4 \%$ of pituitary glandchiasmatic region tumors. ${ }^{5}$ Therefore, the preoperative differentiation of PM from pituitary adenoma based on imaging studies has critical importance.

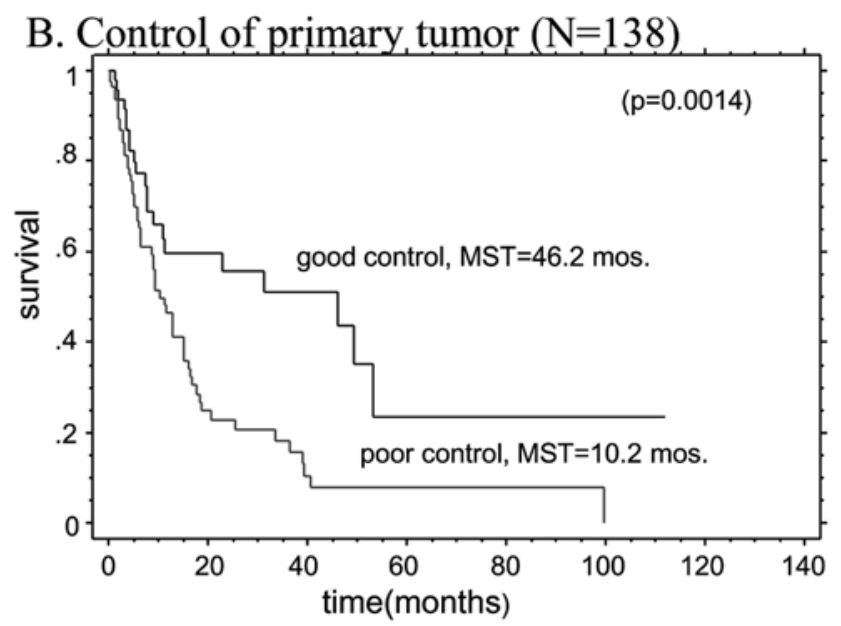

\section{Radiotherapy $(\mathrm{N}=165)$}

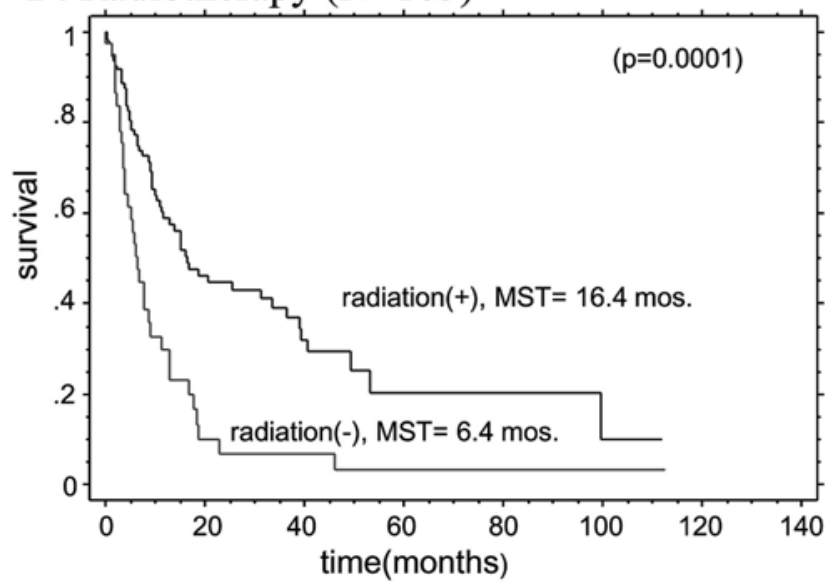

FIG. 10. Survival curve analysis (Kaplan-Meier) according to possible prognostic factors. A: Primary tumors $(n=112)$. B: Control of primary tumor $(n=138)$. C: Patients' age in years $(n=165)$. D: Radiotherapy $(n=165)$. 
TABLE 2. Comparison of clinical features between the definite group and nondefinite group

\begin{tabular}{lcc}
\hline \multicolumn{1}{c}{ Variable } & $\begin{array}{c}\text { Definite } \\
\text { Group }(\mathrm{n}=69)\end{array}$ & $\begin{array}{c}\text { Nondefinite } \\
\text { Group }(\mathrm{n}=132)\end{array}$ \\
\hline Primary tumor & & \\
\hline Lung & $23.2 \%$ & $43.9 \%$ \\
\hline Breast & $23.2 \%$ & $22.7 \%$ \\
\hline Kidney & $7.2 \%$ & $6.8 \%$ \\
\hline Colon/rectum & $10.1 \%$ & $4.5 \%$ \\
\hline Lymphatic & $5.8 \%$ & $4.5 \%$ \\
\hline Unknown & $2.9 \%$ & $5.3 \%$ \\
\hline Interval btwn diagnosis of primary & & \\
tumor \& PM in yrs & & \\
\hline 0-1 & $20.3 \%$ & $37.4 \%$ \\
\hline $1-3$ & $17.4 \%$ & $15.3 \%$ \\
\hline $3-5$ & $7.2 \%$ & $9.2 \%$ \\
\hline 5-10 & $15.9 \%$ & $7.6 \%$ \\
\hline$>10$ & $5.8 \%$ & $2.3 \%$ \\
\hline PM preceded primary lesion & $12.9 \%$ & $6.1 \%$ \\
\hline Unknown & $20.3 \%$ & $22.7 \%$ \\
\hline Mean & $3.5 \pm 4.6$ & $2.0 \pm 3.2 *$ \\
\hline Other intracranial metastatic lesion & $27.5 \%$ & $47.0 \%$ \\
\hline Median survival time in mos & 13.8 & $11.4 \dagger$ \\
\hline * $p=0.0183$, Student t-test. & & \\
\hline $\mathrm{p}=0.895$, Log-rank test. & & \\
\hline
\end{tabular}

Some imaging features suggestive of PM have been reported. Constriction at the diaphragma sellae, documented in $44.7 \%(80 / 179)$ of cases in our series, was reported to be $10 \%$ by Komninos et al. ${ }^{16}$ The difference in incidence seems to be deeply affected by the authors' awareness of this finding. It was seen in all of 4 PMs reported by Schubiger and Haller. ${ }^{26}$ The constriction or dumbbell shape may reflect infiltrative growth of the malignant tumor, which passes through the diaphragmatic hiatus without enlarging it and grows under and above it. Edema along the optic chiasm and tract was first described in a craniopharyngioma case and soon reported to be seen in other sellar lesions including PM but rarely seen in pituitary adenoma. ${ }^{25}$ In this study, it was seen in $11.5 \%$ of cases.

Bony changes, such as erosion of surrounding bony structures and sellar enlargement, are well-known findings. ${ }^{16,20}$ Kistler and Pribram reported that enlargement of the sella precludes the possibility of PM.15 The study showed that the length of inferior extension was significantly smaller in PMs, while the length of upward growth was larger than nonfunctioning pituitary adenomas. A PM may manifest clinical symptoms before enlargement of the sella turcica by rapid growth in an upward direction.

\section{Treatment}

Microscopic or endoscopic transsphenoidal surgery may quickly improve ophthalmological symptoms..$^{20,23}$ However, sufficient decompression may be occasionally hindered by the fibrous component of the tumor or excessive bleeding. ${ }^{7,11,16,21,24,27}$ Considering that progression of
TABLE 3. Hazard ratios of presumptive prognostic factors influencing survival in patients with $\mathrm{PM}^{*}$

\begin{tabular}{lllll}
\hline \multicolumn{1}{c}{ Variable } & $\mathrm{df}$ & Item & $\mathrm{p}$ Value & $\mathrm{HR}$ \\
\hline Age (yrs) & 1 & & 0.0012 & 1.034 \\
\hline Sex (male, female) & 1 & Female & 0.1262 & 0.663 \\
\hline Interval until pituitary metastasis (yrs) & 1 & & 0.0010 & 0.708 \\
\hline Control of primary lesion (good, poor, & 2 & & 0.0507 & \\
$\quad$ unknown) & 1 & Good & 0.5615 & 0.741 \\
& 1 & Poor & 0.3159 & 1.611 \\
\hline Maximum diameter of pituitary lesion & 1 & & $<0.0001$ & 1.205 \\
(mm) & & & & \\
\hline Other intracranial metastasis (present, & 2 & & 0.6464 & \\
$\quad$ no, unknown) & 1 & Present & 0.3977 & 0.677 \\
& 1 & No & 0.3622 & 0.656 \\
\hline Chemotherapy (yes, no) & 1 & Yes & 0.7633 & 0.894 \\
\hline Radiotherapy (yes, no) & 1 & Yes & 0.0370 & 0.522 \\
\hline
\end{tabular}

* Factors and values in boldface are statistically significant $(p<0.05)$.

the pituitary lesion itself was the cause of death in only $13 \%$ of patients, the surgical eradication of the pituitary lesion may not significantly benefit the overall survival. ${ }^{16,20,23}$

Currently, 58 Gamma Knife units are in service in Japan (information from personal contact with distributors). In this series, 59.7\% (120/201) of the patients received stereotactic irradiation. Iwai and colleagues reported that 6 of 7 patients achieved regional control and improvement of symptoms after stereotactic radiotherapy with a marginal dosage of 10-14 Gy (mean 11.9 Gy) using the Gamma Knife. ${ }^{13}$ Kano et al. reported their experience of treating 18 patients who harbored PMs using the Gamma Knife. ${ }^{14}$ Although the overall survival was limited, the lesions were well controlled and new symptoms due to tumor progression were demonstrated in only 3 patients. ${ }^{14}$

Considering limited survival time of the patients, poor general condition, and the noninferiority to conventional multiple fractionated radiation in terms of median survival time (see Survival Analysis), stereotactic radiation may become the first choice of radiation treatment for PMs in cases without multiple intracranial metastases.

\section{Prognosis}

The prognosis for patients with PMs is poor, not because of the location per se but because of the advancing stage of the primary cancer. ${ }^{16,20}$ Furthermore, at the time of diagnosis, more than half of the patients have systemic metastasis. ${ }^{9,11,14,18,20}$ Median survival reported in previous series ranged from 6 to 11 months, ${ }^{11,13,16,18,20}$ survival rarely surpassed 3 years ${ }^{22}$ However, in the series of Morita et al., 3 of 36 patients survived at 5 years following diagnosis. ${ }^{20}$ A very recent report also showed a prolonged median survival time of 11.8 months (ranging from 3 to 43 months) in a series of 15 PMs. ${ }^{31}$ The median survival time was 12.9 months in our series. Given that only $13.2 \%$ of patients died of progression of the pituitary lesion, the improved survival may have been due to development of systemic treatment and management for advanced cancer.

The elongation of the survival will necessitate the change of management strategy for patients with PMs, 
from terminal care to long-term maintenance of quality of life. In this context, appropriate treatment of pituitary dysfunction, including gonadotropin as well as cortisol and thyroxine levels, which is rarely recovered by treatment aimed at the pituitary lesion, is essential. ${ }^{20}$

\section{Limitations of This Study and Future Study}

First, it was surprising that more than $60 \%$ of the patients were diagnosed with PMs based on neuroimaging findings, clinical presentation, clinical course, and an increase in blood tumor markers, or combinations thereof, and not based on histological evidence. In this group, referred to as the nondefinite group, we cannot exclude a possibility that non-PM cases such as pituitary incidentaloma were included in the cohort. However, this group had more biologically aggressive primary tumors such as lung cancer, more advanced cancer stage, and shorter interval until the detection of PM (see Results). These conditions might have led to poorer general condition precluding biopsy surgery in the nondefinite group. Recent reports on PM also include a large proportion of nondefinite group of diagnosis. ${ }^{13,14}$ We now know that many clinicians diagnosed some PMs without histological evidence and managed these cases based on their tentative diagnoses. We need more detailed clinical and imaging data leading to diagnosis of PMs without pathology to get a holistic understanding of nondefinite PMs. Second, we could not include the analyses of patients' performance status and quality of life, which is mandatory to select proper treatment for this serious illness and should be a task in future studies. Third, due to the retrospective nature of this study, there were frequent missing values in clinical and radiological data, which might have distorted the true figures of the PMs. Fourth, we cannot elicit the rate of pituitary metastasis for each kind of malignant neoplasm due to the low response rate to the questionnaire (50\% in hospital base), which may cause considerable selection bias. Furthermore, there is a high probability that many patients with PMs were cared for in small clinics and palliative care centers, to which the questionnaire was not sent, without consulting specialists. Future population-based surveys should be, thus, prospective and inventory.

\section{Conclusions}

An analysis of 201 cases reported as pituitary metastasis in Japan showed that the most frequent primary tumor was lung cancer followed by breast cancer. Almost $60 \%$ of the patients underwent stereotactic radiation for controlling the pituitary lesion. Median survival time was substantially longer, 12.9 months, compared with previous reports. Half of the deaths were caused by progression of the primary lesion, followed by extracranial and intracranial metastases. Only $13.2 \%$ of patients died due to an aggravation of pituitary lesion. Considering that more than half of the patients survive longer than a year, PM patients should not be always subjected to terminal-stage care. Appropriate appraisal of pituitary dysfunction and its pertinent replacement are mandatory. The treatment modality should be elucidated by future studies in the light of survival and better quality of life.

\section{Acknowledgments}

We highly appreciate the physicians who kindly sent case records to us: members of The Japan Endocrine Society, members of The Japanese Society for Hypothalamic and Pituitary Tumors, and doctors belonging to stereotactic radiation centers (see Appendix).

We declare that each of us acknowledges that he or she participated sufficiently in the work to take public responsibility for the content of this paper.

\section{Appendix}

Physicians who reported cases with pituitary metastasis: Kosaku Amano, Daisuke Aono, Noriaki Fukuhara, Noriaki Hanyu, Hironobu Harada, Yuji Hataya, Michio Hayashi, Motohiro Hayashi, Yasuhiko Hayashi, Hisao Hirai, Kentaro Horiguchi, Kaoru Ichihara, Hidetoshi Ikeda, Chie Inomoto, Akihiro Inoue, Yasuhi Inoue, Kenichi Ishibashi, Eiji Itagaki, Miiko Ito, Toshihiko Iuchi, Yoshiyasu Iwai, Naoko Iwata, Hidefumi Jokura, Teruyoshi Kageji, Wataru Kameda, Tadashige Kano, Junko Kato, Toshiro Katsuta, Jun Kawagishi, Takakazu Kawamata, Hirotsugu Kawamoto, Ryogo Kikuchi, Toshikazu Kimura, Yasuyuki Kinoshita, Atsushi Kiyota, Ryuhei Kitai, Yasato Komatsu, Takeshi Kondo, Atsushi Kuge, Kazuhiko Kurozumi, Hiroyuki Masaoka, Yuka Matoba, Akira Matsuno, Takayuki Matsuo, Misaki Miyata, Katsuharu Mori, Yoshimasa Mori, Ryo Morikawa, Tetsuya Nagatani, Yasushi Nagatomo, Mizuho Nakagawa, Takuo Nanbu, Shigeki Nishino, Ryutaro Nomura, Koichiro Ogawa, Hisayo Okamoto, Kazuya Okamoto, Takahiro Okusu, Hiroaki Onishi, Naokatsu Saeki, Yoshinori Saika, Katsuya Saito, Keiichi Sakai, Tetsuro Sameshima, Kengo Sato, Masahiro Shin, Shuji Shinkawa, Junji Shinoda, Hitoshi Sugihara, Nami Suzuki, Satoshi Suzuki, Shigeyuki Tahara, Masashi Takagi, Shingo Takano, Yoshiyu Takeda, Susumu Takekoshi, Nobuo Takenaka, Masahi Tamaki, Tetsuro Tamura, Koshi Tatewaki, Kinya Terada, Yuzo Terakawa, Masahiro Toda, Masahiko Tosaka, Nagaoki Toyoda, Kumiko Tsuboi, Masashi Uchida, Hiroki Udono, Tetsuya Ueba, Kenta Ujifuku, Kiyoaki Uryu, Takeshi Usui, Masayo Yamada, Hideki Yamaguchi, Junkou Yamamoto, Tetsuo Yamashita, Shigetoshi Yano, Soichiro Yasuda, Kazutaka Yatsushiro, Tadato Yonekawa, Yuichiro Yoneoka, Mitsuhiro Yoneya, Masanori Yoshida, Miyako Yoshizawa.

\section{References}

1. Bakhtiar Y, Hanaya R, Tokimura H, Hirano H, Oyoshi T, Fujio S, et al: Geometric survey on magnetic resonance imaging of growth hormone producing pituitary adenoma. Pituitary 17:142-149, 2014

2. Bellew S, Del Rosso JQ, Kim GK: Skin cancer in Asians: part 2: melanoma. J Clin Aesthet Dermatol 2:34-36, 2009

3. Branch CL Jr, Laws ER Jr: Metastatic tumors of the sella turcica masquerading as primary pituitary tumors. J Clin Endocrinol Metab 65:469-474, 1987

4. Chiang MF, Brock M, Patt S: Pituitary metastases. Neurochirurgia (Stuttg) 33:127-131, 1990

5. Committee of the Brain Tumor Registry of Japan: Report of Brain Tumor Registry of Japan (1984-2000). Neurol Med Chir (Tokyo) 49 Suppl:PS1-PS96, 2009

6. Duchen LW: Metastatic carcinoma in the pituitary gland and hypothalamus. J Pathol Bacteriol 91:347-355, 1966

7. Fassett DR, Couldwell WT: Metastases to the pituitary gland. Neurosurg Focus 16(4):E8, 2004

8. Freda PU, Post KD: Differential diagnosis of sellar masses. Endocrinol Metab Clin North Am 28:81-117, vi, 1999

9. Gsponer J, De Tribolet N, Déruaz JP, Janzer R, Uské A, Mirimanoff RO, et al: Diagnosis, treatment, and outcome of pituitary tumors and other abnormal intrasellar masses. Retrospective analysis of 353 patients. Medicine (Baltimore) 78:236-269, 1999 
10. Hägerstrand I, Schönebeck J: Metastases to the pituitary gland. Acta Pathol Microbiol Scand 75:64-70, 1969

11. Houck WA, Olson KB, Horton J: Clinical features of tumor metastasis to the pituitary. Cancer 26:656-659, 1970

12. Hsing AW, Tsao L, Devesa SS: International trends and patterns of prostate cancer incidence and mortality. Int J Cancer 85:60-67, 2000

13. Iwai Y, Yamanaka K, Honda Y, Matsusaka Y: Radiosurgery for pituitary metastases. Neurol Med Chir (Tokyo) 44:112 117,2004

14. Kano H, Niranjan A, Kondziolka D, Flickinger JC, Lunsford LD: Stereotactic radiosurgery for pituitary metastases. Surg Neurol 72:248-256, 2009

15. Kistler M, Pribram HW: Metastatic disease of the sella turcica. Am J Roentgenol Radium Ther Nucl Med 123:13-21, 1975

16. Komninos J, Vlassopoulou V, Protopapa D, Korfias S, Kontogeorgos G, Sakas DE, et al: Tumors metastatic to the pituitary gland: case report and literature review. J Clin Endocrinol Metab 89:574-580, 2004

17. Kovacs K: Metastatic cancer of the pituitary gland. Oncology 27:533-542, 1973

18. Max MB, Deck MD, Rottenberg DA: Pituitary metastasis: incidence in cancer patients and clinical differentiation from pituitary adenoma. Neurology 31:998-1002, 1981

19. McCormick PC, Post KD, Kandji AD, Hays AP: Metastatic carcinoma to the pituitary gland. Br J Neurosurg 3:71-79, 1989

20. Morita A, Meyer FB, Laws ER Jr: Symptomatic pituitary metastases. J Neurosurg 89:69-73, 1998

21. Nelson PB, Robinson AG, Martinez AJ: Metastatic tumor of the pituitary gland. Neurosurgery 21:941-944, 1987

22. Ntyonga-Pono MP, Thomopoulos P, Luton JP: [Pituitary metastases. 3 cases.] Presse Med 28:1567-1571, 1999 (Fr)

23. Pinet C, Raholimina V, Ferri RM, Kleisbauer JP: [Panhypopituitarism secondary to pituitary metastases.] Presse Med 29:17-18, 2000 (Fr)

24. Ruelle A, Palladino M, Andrioli GC: Pituitary metastases as presenting lesions of malignancy. J Neurosurg Sci 36:51-54, 1992
25. Saeki N, Murai H, Kubota M, Fujimoto N: Oedema along the optic tracts due to pituitary metastasis. Br J Neurosurg 15:523-526, 2001

26. Schubiger O, Haller D: Metastases to the pituitary-hypothalamic axis. An MR study of 7 symptomatic patients. Neuroradiology 34:131-134, 1992

27. Sioutos P, Yen V, Arbit E: Pituitary gland metastases. Ann Surg Oncol 3:94-99, 1996

28. Teears RJ, Silverman EM: Clinicopathologic review of 88 cases of carcinoma metastatic to the pituitary gland. Cancer 36:216-220, 1975

29. Troisi R, Altantsetseg D, Davaasambuu G, Rich-Edwards J, Davaalkham D, Tretli S, et al: Breast cancer incidence in Mongolia. Cancer Causes Control 23:1047-1053, 2012

30. Yasuda A, Campero A, Martins C, Rhoton AL Jr, Ribas GC: The medial wall of the cavernous sinus: microsurgical anatomy. Neurosurgery 55:179-190, 2004

31. Zoli M, Mazzatenta D, Faustini-Fustini M, Pasquini E, Frank G: Pituitary metastases: role of surgery. World Neurosurg 79:327-330, 2013

\section{Author Contributions}

Conception and design: Hirano, Habu, Tokimura, Yunoue, Shimatsu, Teramoto, Arita. Acquisition of data: Habu, Tokimura, Yasuda, Nagatomo, Iwai, Kawagishi, Tatewaki, Yunoue, Campos, Kinoshita, Arita. Analysis and interpretation of data: Hirano, Tokimura, Yunoue, Campos, Kinoshita. Drafting the article: Hirano, Habu, Shimatsu, Arita. Critically revising the article: Hirano, Habu. Reviewed submitted version of manuscript: Hirano. Approved the final version of the manuscript on behalf of all authors: Hirano. Statistical analysis: Hirano, Yunoue. Administrative/technical/material support: Habu, Shimatsu. Study supervision: Hirano, Shimatsu, Teramoto, Arita.

\section{Correspondence}

Hirofumi Hirano, Department of Neurosurgery, Graduate School of Medical and Dental Sciences, Kagoshima University, 8-35-1, Sakuragaoka, Kagoshima, 890-8520, Japan. email: hirahira@ m2.kufm.kagoshima-u.ac.jp. 